\title{
Access to Lexical Information in Language Comprehension: Semantics before Syntax
}

\author{
Oliver Müller ${ }^{1,2}$ and Peter Hagoort ${ }^{2,3}$
}

\begin{abstract}
The recognition of a word makes available its semantic and syntactic properties. Using electrophysiological recordings, we investigated whether one set of these properties is available earlier than the other set. Dutch participants saw nouns on a computer screen and performed push-button responses: In one task, grammatical gender determined response hand (left/right) and semantic category determined response execution (go/no-go). In the other task, response hand depended on semantic category, whereas response execution depended on gender. During the latter task, response preparation oc-
\end{abstract}

\section{INTRODUCTION}

Reading a text is an enterprise that begins with the visual perception of lines and dots and ends with some notion about the meaning of the text. One major step in this process is word recognition, that is, identifying a configuration of script symbols as a particular word. In the course of word recognition, lexical knowledge-the meaning and syntactic properties of a word-becomes available. This forms the input to sentence processing, which assembles the information associated with individual words into larger syntactic and semantic structures. The access to semantic and syntactic word properties is an important transition point. By proceeding from the word as perceptual unit to the word as carrier of semantic and syntactic information, it becomes possible to (re)construct a meaningful message from a string of words. Word meanings form as it were the building blocks of overall text meaning, whereas syntactic word properties give information about how words relate to each other. The grammatical gender of nouns is a syntactic word property, which exists in a wide variety of languages, such as Dutch, Italian, Hebrew, and Swahili (cf. Corbett, 1991). Depending on the language at hand, grammatical gender of a noun can determine the form

\footnotetext{
${ }^{1}$ Max Planck Institute for Psycholinguistics, Nijmegen, The Netherlands, ${ }^{2}$ F.C. Donders Centre for Cognitive Neuroimaging, Nijmegen, The Netherlands, ${ }^{3}$ Nijmegen Institute for Cognition and Information, Radboud University Nijmegen, The Netherlands
}

curred on no-go trials, as measured by the lateralized readiness potential: Semantic information was used for response preparation before gender information inhibited this process. Furthermore, an inhibition-related N2 effect occurred earlier for inhibition by semantics than for inhibition by gender. In summary, electrophysiological measures of both response preparation and inhibition indicated that the semantic word property was available earlier than the syntactic word property when participants read single words.

of articles, adjectives, pronouns, and other kind of words, a phenomenon called gender agreement. The Dutch language knows two grammatical genders, labeled common and neuter gender. In Dutch, the most prominent instance of gender agreement concerns the form of the singular definite article. For common gender nouns, the article takes the form $d e$ and for neuter gender nouns, it takes the form het. Translating The farmer caught the lamb into Dutch results in De boer pakte het lam, because boer is of common gender and lam is of neuter gender.

Despite the importance of the access to semantic and syntactic word properties, little is known about the time course of the involved retrieval operations. In this article, we want to focus on the relative time course regarding the availability of semantic and syntactic properties of single words. Is lexico-semantic information available before lexico-syntactic information, or is the opposite true? Although numerous studies investigated the impact of semantic and syntactic context on word recognition, they focused on whether and how this might occur, often treating semantic and syntactic contexts separately (Friederici \& Jacobsen, 1999; Balota, 1994). And although the field of sentence processing figures a controversy about when syntactic and semantic information are used in parsing (e.g., Friederici, 2002; MacDonald, Pearlmutter, \& Seidenberg, 1994; Trueswell \& Tanenhaus, 1994; Frazier, 1989), an extrapolation from sentence processing to retrieval of semantic and syntactic word properties during word recognition seems not straightforward. 
In speech production research, clear assumptions about the time course of access exist. A wide range of models assumes that word properties are represented in an ordered set of layers, with activation spreading from a semantic layer to a syntactic layer (the so-called lemma layer) and from there to a phonological layer (cf. Levelt, 1999; but see Caramazza, 1997). Thus, semantic and syntactic aspects of words are represented in separate layers, with semantic word properties receiving activation before syntactic word properties. ${ }^{1}$ Levelt, Roelofs, and Meyer (1999) have proposed that language comprehension and production share the semantic and the lemma level: "the perceptual and production networks coincide from the lemma level upwards" (p. 7). One can assume that the direction of processing in comprehension is reversed in relation to that in speech production. Whereas processing in comprehension starts with a perceived word shape and heads towards a conceptual understanding, in production it starts with a conceptual intention and heads towards word shape. Regarding the reading of words, one would then expect syntactic word properties to be available earlier than semantic properties.

Here, we investigated the time course of syntactic and semantic activation in single word reading by means of specific brain responses, the so-called lateralized readiness potential (LRP) and the inhibition-related N2, taking advantage of the temporal resolution of a few milliseconds characteristic of event-related brain potentials (ERPs). The LRP is regarded an index of specific response preparation and is derived from the readiness potential (RP), which can be observed before voluntary hand movements (Kornhuber \& Deecke, 1965). Kutas and Donchin (1980) showed that the RP for unimanual responses is largest at electrodes above the motor cortex contralateral to the moving hand, corresponding to the functional neuroanatomy of movement preparation. The LRP represents the average lateralization of movementrelated activity and indicates that response preparation for a particular hand takes place (Coles, Gratton, \& Donchin, 1988; De Jong, Wierda, Mulder, \& Mulder, 1988). In combination with the two-choice go/no-go paradigm, the LRP has proven useful to investigate the temporal dissociation of different kinds of information (Miller \& Hackley, 1992; Osman, Bashore, Coles, Donchin, \& Meyer, 1992).Van Turennout, Hagoort, and Brown (1997) pioneered in applying this paradigm to the investigation of time-course issues in language processing. They studied the access to semantic and phonological word properties in speech production. Participants performed a picture-naming task in Dutch. On some trials, they additionally had to perform a twochoice go/no-go task: The semantic category of the pictures (animal/object) determined whether to give a response with the left or right hand, whereas the last phoneme of the picture name determined whether a response should be actually executed or not (go/no-go).
As semantic information would become available, the movement of a particular hand could be prepared. That in turn should appear as lateralized motor activity, that is, an LRP. Most interestingly, Van Turennout et al. found temporary LRP activity in the no-go-condition, which declined again without triggering an overt response. Thus, semantic information instructed specific response preparation for some time before phonological information stopped this process. This suggests that semantics is available before phonology in speech production. However, Smid, Mulder, Mulder, and Brands (1992) demonstrated that participants can exert strategic control about the order in which they use stimulus information in the two-choice go/no-go paradigm. In particular, participants might prefer to use the response hand information first and the go/no-go information later, in order to start movement preparation as soon as possible and gain faster responses on go-trials. This could have been an alternative explanation of the no-go-LRP activity in Van Turennout et al.'s experiment, without reference to some intrinsic processing order. However, this strategic account would also predict a no-go-LRP under reversed instructions, with phonology determining response hand and semantics determining the go/no-go decision. Van Turennout et al. conducted a second experiment with exactly these instructions, but found no LRP for nogo-trials, ruling out the strategic account.

Schmitt, Münte, and Kutas (2000) introduced another ERP component for monitoring the time course of language processing, the inhibition-related N2. In experiments with a go/no-go task, the ERP for no-go-trials displays a negative peak of several microvolts with a frontocentral distribution (Thorpe, Fize, \& Marlot, 1996; Gemba \& Sasaki, 1989; Kok, 1986; Pfefferbaum, Ford, Weller, \& Kopell, 1985; Simson, Vaughan, \& Ritter, 1977). It is widely assumed that the no-go-related negativity reflects response inhibition (Falkenstein, Hoormann, \& Hohnsbein, 1999; Eimer, 1993; Jodo \& Kayama, 1992; Kok, 1986). Schmitt et al. (2000) employed the inhibitionrelated N2 in a study that replicated Van Turennout et al. (1997) in German. Semantics and phonology of picture names again determined response hand and the go/no-go decision. When semantics controlled the inhibition-relevant go/no-go decision, the N2 effect occurred significantly earlier than when phonology did. This again provided evidence for the earlier availability of semantic information relative to phonological information. The LRP analysis corroborated the N2 finding, paralleling the results of Van Turennout et al.

Schmitt, Rodriguez-Fornells, Kutas, and Münte (2001) applied the two-choice go/no-go paradigm to semantic and syntactic access, during both speech production and auditory language comprehension. They employed an animal/object categorization tapping into semantic processing and a (German) grammatical gender categorization tapping into syntactic processing. The speech production experiment used picture stimuli, whereas 
the auditory comprehension experiment used sound recordings of the picture names. In each experiment, semantic category and gender were alternately mapped onto response hand and the go/no-go decision. In the speech production experiment, the N2 occurred earlier when semantics determined response inhibition than when gender did. This concurs with the assumptions of speech production theories. It is furthermore supported by a behavioral picture-naming study that manipulated the semantic relatedness and gender congruency of distractor words at several SOAs (Schriefers \& Teruel, 2000). Effects of semantic relatedness occurred at earlier SOAs than effects of gender congruency. Schmitt, Rodriguez-Fornells, et al.'s second ERP experiment, with auditorily presented words, showed the same retrieval order as for pictures, that is, an earlier N2 effect for semantics than for gender. This is actually the opposite outcome one expects under the assumption that speech production and language comprehension share the representations of semantic and syntactic word properties, but access them in a reversed order.

However, one might hesitate to generalize timecourse findings from an experiment with spoken words to the processing of written words. The spoken word form is extended in time and information from the unfolding speech signal is assumed to be continuously mapped onto lexical representations (Norris, 1994; Marslen-Wilson, 1987; McClelland \& Elman, 1986). In contrast, the written word form provides all perceptual information at once and, at least in principle, information from all letter positions could be processed in parallel (cf. Zorzi, 2000; Rastle \& Coltheart, 1999; Radeau, Morais, Mousty, Saerens, \& Bertelson, 1992). Should cues to different kinds of information (e.g., semantic and syntactic) be located at separate positions of a word form, this would entail in the auditory modality that these cues would appear at different points in time. That is, information extractable from an early position would have a temporal advantage over information at a late position. Allopenna, Magnuson, and Tanenhaus (1998) have provided evidence that listeners actually pick up information in the order in which it becomes available in the speech signal. They used spoken stimuli such as beaker, which is consistent with beetle in its initial portion and with speaker in its later portion. Both these partially consistent words showed signs of activation in a continuous eye movement measure, but the onset-related beetle (and beaker itself) showed an earlier effect than the rhyming word speaker. Thus, the effect indicating the word's activation mirrored the time course of the acoustic evidence for that word. A study by Van den Brink and Hagoort (2004) (also see Hagoort, 2003) indicates that also semantic and syntactic information of a spoken word are extracted in the order in which corresponding cues occur in the speech signal. In an ERP experiment, they presented sentences where the critical word was either semanti- cally appropriate or inappropriate and the inappropriate word additionally violated lexical category constraintsbeing a verb although a noun was required. The semantic violation could be detected relatively early, as the phonological form of the inappropriate item deviated from the most expected word. Concerning the syntactic violation, the incoming speech signal was consistent with the inappropriate word being a noun until the last syllable, which was a past tense inflectional suffix identifying the word as a verb. This was deliberately different from earlier auditory studies, where a prefix had marked the lexical category violation right at word onset (e.g., Hahne \& Jescheniak, 2001; Friederici, Pfeifer, \& Hahne, 1993). In the latter experiments, lexical category violations elicited a left anterior negativity earlier than the usual timing of the semantic N400 effect (cf. Kutas \& Van Petten, 1994). However, this order was reversed in the Van den Brink and Hagoort study where lexical category information occurred late in the word. Thus, it seems that also the availability of semantic and syntactic information depends on their temporal position within the spoken word form.

The interpretation of time-course findings obtained with spoken words should therefore take into account that cues to semantic and syntactic information could have occurred in temporally different stretches of words and that such signal-related availability could have biased the findings. An experiment with written words, where all perceptual information is delivered simultaneously, would not be prone to such an influence and might lead to different time-course results. Therefore, we decided to conduct an experiment with written word forms to investigate the time course with which semantic and syntactic information becomes available in language comprehension.

Participants performed a two-choice go/no-go task on single Dutch nouns, with a semantic category decision and a grammatical gender decision probing the access to semantic and syntactic word properties. One-half of the participants received instructions in which grammatical gender determined response hand (left/right) and semantic category determined the go/no-go decision. The other half received instructions that mapped semantic category on response hand and grammatical gender on the go/no-go decision (see Table 1 for examples).

\section{RESULTS}

The data analysis includes only words of common gender. About half of our neuter gender words were diminutives. They are marked by an orthographic suffix and automatically possess neuter gender in Dutch. In this way, de kerk (the church) can become het kerkje (several allomorphs are used, depending on the phonological form of the base word, but all end in -je; cf. Donaldson, 1987). Inclusion of the diminutives in the material had been necessary to arrive at an approximate- 
Table 1. Example of the Mapping of the Stimulus Dimensions Grammatical Gender and Semantic Category onto the Response Dimensions Hand and Response Execution, with Dutch Example Words (English Translation in Brackets)

\begin{tabular}{lcc}
\hline Task: hand $=$ semantics, go/no-go $=$ gender \\
\hline common $=$ go & neuter $=$ no-go \\
\hline building $=$ left hand & schuur (barn) & hotel (hotel) \\
consumable = right hand & melk (milk) & zout (salt) \\
Task: hand = gender, go/no-go $=$ semantics \\
\hline \multicolumn{4}{c}{ common = left } & neuter $=$ right \\
hand & hand \\
\hline building $=$ go & schuur (barn) & hotel (hotel) \\
consumable = no-go & melk (milk) & zout (salt) \\
\hline
\end{tabular}

ly equal amount of common and neuter gender words in each semantic category. Finding enough words with intrinsic neuter gender was complicated by the fact that Dutch shows a preponderance towards common gender, which makes up about three quarter of all nouns (cf. Van Berkum, 1996). Because of the orthographic marking, diminutives are not suited to investigate lexical access to grammatical gender, and the number of intrinsically neuter gender items was insufficient for a separate analysis of their electrophysiological data.

We recorded the electromyogram (EMG) of the index fingers and discarded no-go-trials if they showed muscular activity and go-trials if they showed activity of the wrong response hand (if not already excluded as incorrect overt response). In this way, we controlled for low-level muscle activity on no-go-trials indicating incomplete inhibition, which is an unwanted source of temporary LRP activity. Concerning the go-conditions, activation of both hands would corrupt the go-LRP. Trials showing eye movement or EEG artifacts were also excluded from all analyses, whereas trials with behavioral errors were excluded from RT and ERP analyses.

\section{Overt Responses}

The RT for common gender words averaged across participants was $775 \mathrm{msec}$ for the hand = semantics task $(\mathrm{go} / \mathrm{no}-\mathrm{go}=$ gender $)$ and $796 \mathrm{msec}$ for the hand $=$ gender task $(\mathrm{go} / \mathrm{no}-\mathrm{go}=$ semantics). The difference between the two tasks did not reach significance in a $t$ test, with $t(30)=-1.1, S E=19.8$, and $p=.291$.

Different types of error apply to go-trials (wrong response hand and timeout) and no-go-trials (any overt response). Therefore, we performed separate error analyses for the go- and no-go-conditions. The average error percentage on go-trials was 3.2 for the hand $=$ semantics task (go/no-go $=$ gender) and 5.4 in the hand $=$ gender task $(\mathrm{go} /$ no-go $=$ semantics $)$. This was significant in a $t$ test, with $t(30)=-2.2, S E=0.966$, and $p=.037$. For no-go trials, the average error percentage was 0.7 in the hand $=$ semantics task and 0.8 in the hand $=$ gender task. The difference was not significant $[t(30)=-0.2, S E=0.430, p=.833]$.

\section{Lateralized Readiness Potential}

We computed stimulus-locked LRPs for the hand $=$ semantics task (go/no-go $=$ gender) and the hand $=$ gender task (go/no-go $=$ semantics), separately for the go and no-go conditions. ${ }^{2}$ The reported results are based on waveforms which represent the average of LRPs from the electrode pairs $18 / 10$ and $7 / 3$. Inspection of participant and grand-average waveforms had shown that they provided the biggest go-LRP amplitude. The averaging combined the LRP data from both electrodes into one dependent measure, so that activations consistently present at both electrodes were favored in the analysis. To test for correct preparatory motor activity in the LRP, we performed series of $t$ tests on voltage amplitude. First, moving averages of voltage were computed for the go- and no-go-conditions of both tasks, derived from all subject LRPs. Window width was $50 \mathrm{msec}$, with the first window starting at $6 \mathrm{msec}$ and ending at $54 \mathrm{msec}$ after stimulus onset, so that its center was at $30 \mathrm{msec}$. The next window was shifted in time by $10 \mathrm{msec}$, having its center at $40 \mathrm{msec}$ and so forth. The last window had its center at $1000 \mathrm{msec}$ after stimulus onset. For every window, a one-tailed $t$ test against zero was performed on the moving averages. If five consecutive windows with negative voltage had a $p$ value $<.05$, we assumed that a meaningful deviation from baseline had occurred, that is, some correct preparatory activation had been present. The center of the first significant window counted as onset latency of LRP activity.

Grand-average LRPs for the go and no-go condition of the hand $=$ semantics task $(\mathrm{go} /$ no-go $=$ gender $)$ are shown in Figure 1A. Go- and no-go-waveform showed no significant activity during the first $400 \mathrm{msec}$. At $440 \mathrm{msec}$, the go LRP started to deviate significantly from baseline $[-11.8<t(15)<-1.7, .001<p<.047]$. Significant no-go-activity occurred shortly later, from 480 to 530 msec $[-2.6<t(15)<-1.7, .012<p<.05]$ and from 570 to 620 msec $[-2.5<t(15)<-1.7, .014<p<.043]$. Go and no-go waveform ran parallel during the ascent from baseline, whereas later the go-LRP continued to increase and the no-go LRP entered a gradual decline to baseline. We compared the two waveforms directly with a series of paired-samples $t$ tests on the moving averages, which yielded no significantly different region until 510 msec [from then on: $-7.7<t(15)<-2.1, .001<p<$ .05 ; criterion of five consecutive significant windows].

For the hand $=$ gender task (go/no-go $=$ semantics), grand-average LRPs are presented in Figure 1B. The go-LRP displayed some early significant activity from 
Figure 1. LRP grand averages for go- and no-go-trials, averaged across the electrode pairs 18/10 and $7 / 3$. Arrows mark the average RT for the go-conditions of the two tasks. (A) Task hand $=$ semantics, rectangle indicates the period from the onset of no-go-activity $(480 \mathrm{msec})$ to the divergence of go- and no-go-waveform $(510 \mathrm{msec}$ ). (B) Task hand $=$ gender, go/ no-go $=$ semantics. go/no-go $=$ gender. The gray

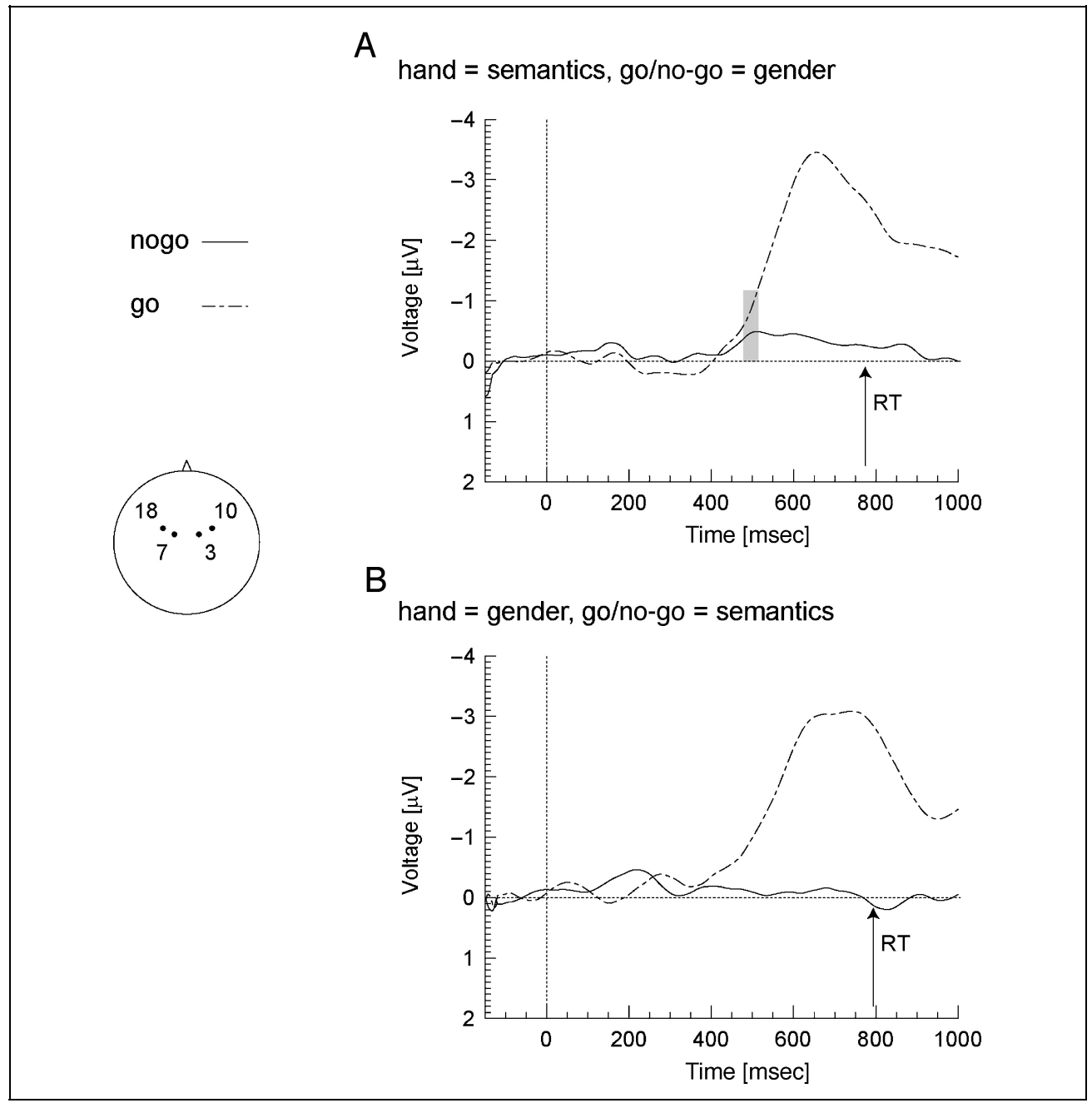

260 to 310 msec $[-2.6<t(15)<-1.9, .011<p<.034]$, whereas the main negativity started to deviate significantly from baseline at $400 \mathrm{msec}[-8.1<t(15)<-1.7$, $.001<p<.045]$. In the no-go-condition, there was significant activity from 140 to $260 \mathrm{msec}[-3.7<$ $t(15)<-1.8, .001<p<.039]$. No other regions showed significant deviations from baseline.

Additionally, we tested whether the onset of the goLRP main negativities differed between tasks. We took the moving averages of both go LRPs and compared them through a series of two-tailed independentsamples $t$ tests. The go LRPs differed significantly only between 260 and $310 \mathrm{msec}[2.3>t(30)>2.1, .029<$ $p<.049$ ]. From $340 \mathrm{msec}$ on, all $p$ values $>.10 .^{3}$

\section{N2 Inhibition-related Effect}

We computed participant and grand averages for the go and no-go conditions of the go/no-go = gender and the go/no-go $=$ semantics task. In both tasks, the go and no-go waveforms developed in parallel during the first $250 \mathrm{msec}$. At frontal electrodes, a negative peak around 125 msec was followed by a positive peak around
175 msec after stimulus presentation (see Figure 2A and $\mathrm{B}$ ). Then a sustained negativity with a peak around $450 \mathrm{msec}$ appeared for no-go-conditions, diverging from the go-waveform at roughly $350 \mathrm{msec}$ for the gender = go/no-go task and at $250 \mathrm{msec}$ for the semantics $=\mathrm{go} /$ no-go task. To obtain a net inhibition effect, we subtracted the go conditions from the no-go conditions. The subtraction waveforms displayed a negative effect, comparable to the N2 effects reported in earlier ERP studies using inhibition paradigms (Figure 2C). The most noticeable difference between the N2 effects of the two tasks was that the effect for the go/no-go = semantics task seemed to occur earlier than the effect for the go/no-go = gender task.

We determined the peak latency of the N2 effect by searching for the largest negative voltage value within a time window from 300 to $700 \mathrm{msec}$ after stimulus onset, separately for all 10 frontal electrodes. An ANOVA was performed on the peak latencies, with Task as betweensubjects factor and Electrode as within-subjects factor. ${ }^{4}$ The peak latencies across electrodes and participants were $454 \mathrm{msec}$ for the go/no-go = semantics task and 554 for the go/no-go = gender task. The corresponding 
Figure 2. Grand averages of the N2 inhibition-related effect, averaged across all 10 frontal electrodes indicated in the head schema. (A) No-go- and go-trials in the go/no-go $=$ gender task. (B) No-go- and go-trials in the go/no-go $=$ semantics task. (C) N2 net inhibition effect for both tasks, resulting from the subtraction of the no-go- and go-waveforms from (A) and (B).

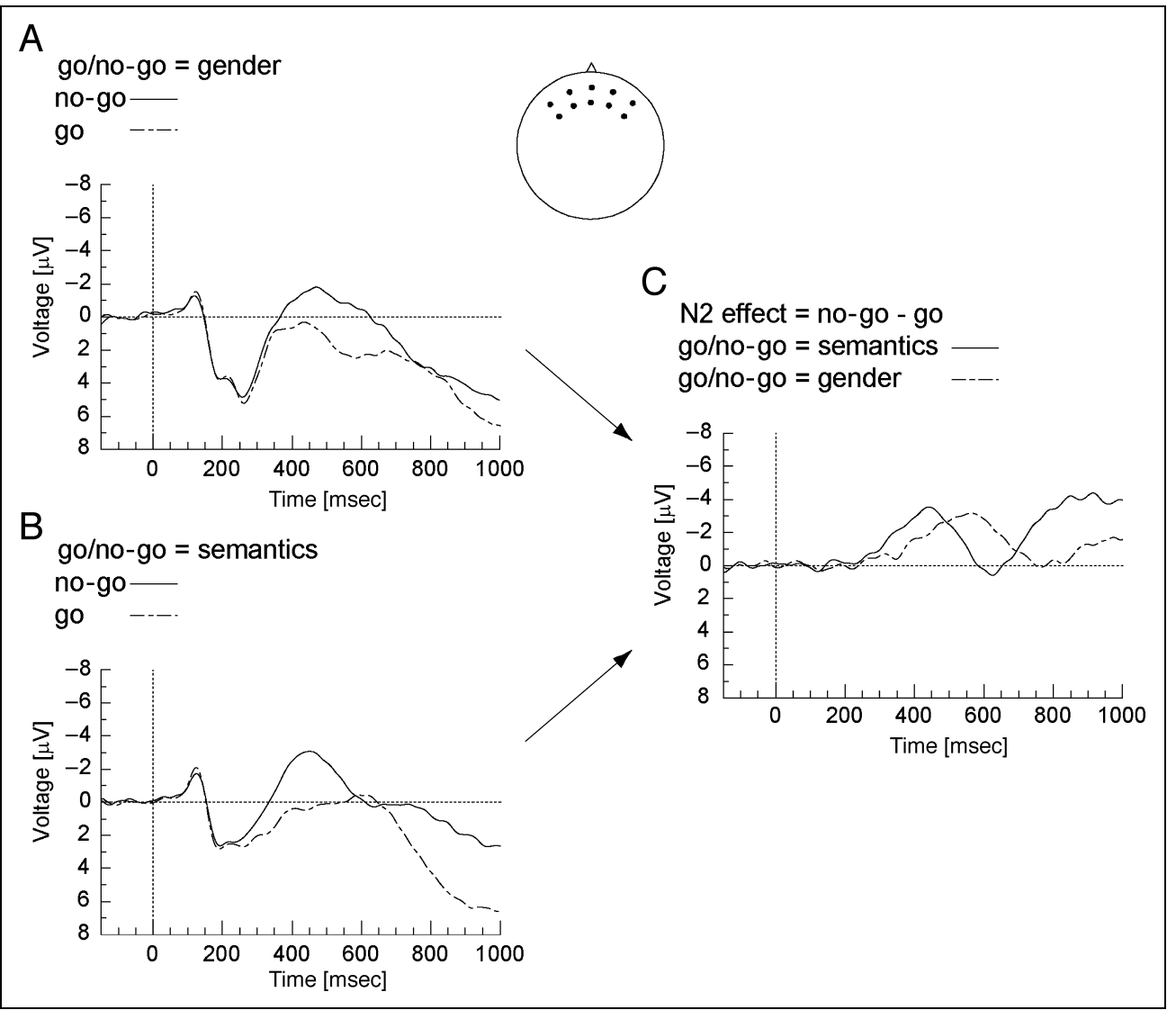

main effect of Task was significant $[F(1,30)=15.0$, $M S E=53771.9$, and $p=.001]$. However, neither the main effect of Electrode $[F(9,270)=1.6, M S E=3523.9$, $p=.179, \varepsilon=.492$ ) nor the interaction between Task and Electrode reached significance $[F(9,270)=1.3$, $M S E=3523.9, p=.270, \varepsilon=.492]$.

\section{DISCUSSION}

In this study, we examined the temporal order, in which semantic category and grammatical gender information become available during the reading of single Dutch words. Two groups of participants performed different two-choice go/no-go tasks, both combining a semantic with a gender classification. For the group carrying out the hand $=$ semantics task (go/no-go $=$ gender), we found some temporary LRP activity in the no-go-condition from 480 to 530 msec. This occurred in parallel with the go-LRP of the same task, which had an onset around $440 \mathrm{msec}$. The group performing the hand = gender task (go/no-go = semantics) showed early LRP activity, from 140 to $260 \mathrm{msec}$ in the no-go condition and from 260 to $310 \mathrm{msec}$ in the go condition. This was well before onset of the main go LRP in that task, which occurred around $400 \mathrm{msec}$. Although onset of the main go LRP seemed to occur somewhat later in the hand = semantics task (440 msec) than in the hand = gender task (400 msec), this difference was statistically not significant. The peak latency for the inhibition-related $\mathrm{N} 2$ effect in the go/no-go = semantics task was $100 \mathrm{msec}$ earlier than in the go/no-go = gender task.

The significant LRP activity in the no-go-condition of the hand $=$ semantics task $(\mathrm{go} / \mathrm{no}-\mathrm{go}=$ gender $)$ indicates that semantic information was available before gender information. In this task, when semantic information becomes available, response preparation for the correct hand can begin. This in turn would surface as LRP activity. The LRP activity starting at $480 \mathrm{msec}$ implies, thus, that semantic information was available at that time. We can also infer that response inhibition, on the basis of gender information, has not set in yetotherwise we should see no LRP activity at all. In other words, semantic information seems to have had an earlier influence on response preparation than gender information. This could reflect the retrieval order of these two word properties in language comprehension. Alternatively, it could reflect some strategic ordering in the use of information. Smid et al. (1992) pointed out that participants might give priority to information that is relevant for motor preparation (in this task, semantics) in order to gain fast responses. Information concerning execution/inhibition (here, gender) would be processed afterwards, as successful inhibition can be instantiated also at a later time. If such a strategic account was true, the particular word properties determining response hand and inhibition should be irrelevant for the occurrence of 
a no-go LRP. Hence, we should find it also for the hand $=$ gender $(\mathrm{go} /$ no-go $=$ semantics $)$ task. Although we found early LRP activity in this task for both the go and no-go condition (treated in the next paragraph), no significant activity in the no-go LRP occurred in parallel to the main go LRP. As argued above, occurrence of LRP activity implies that the information determining response hand was available, which in this task is grammatical gender. The main go LRP started around $400 \mathrm{msec}$ and increased continuously until triggering an overt response. This suggests that gender was available in a reliable manner during this period. It seems reasonable to assume that this was also the case in the no-go condition. However, we found no significant LRP activity for the no-go condition around 400 msec or later. The absence of a no-go LRP in this period suggests that response inhibition, on the basis of semantic category information, was already in effect. This is in contradiction to the strategic account mentioned above, postulating that response preparation should occur before inhibition. It is rather in line with a language-related interpretation, which assumes that semantics is retrieved before gender.

A critical point regarding the interpretation of the LRP data is the early activation found for the go and no-go condition of the hand $=$ gender task. Well before the onset of the main negativity, some correct movement preparation seems to have occurred (go LRP: 260 to $310 \mathrm{msec}$; no-go LRP: 140 to $260 \mathrm{msec}$ ). We would suggest that this is an artifact caused by the presence of diminutives in the materials, which always possess neuter gender and are orthographically marked by a suffix (e.g., kerk-je). For diminutives, the suffix would obviously allow a gender decision on an orthographic basiswhich is why they were excluded from analysis. However, there might even be consequences for the common gender words, which did enter analysis. In every experimental block, there were about one-quarter diminutives, one-quarter intrinsically neuter gender nouns, and one-half common gender nouns. Accordingly, from all words not carrying the diminutive suffix, two-thirds were of common gender. Participants might have used this regularity for a guess, categorizing words without the diminutive suffix as probably having common gender. For common gender words, this would have actually resulted in a correct guess (although not for unmarked neuter words). The guessed gender status then could have led to some correct preliminary motor preparation like we see in the presented data. Because there were also neuter gender words without the diminutive suffix, this strategy was not fail-safe and for accurate performance it was necessary to access lexically stored gender information. Therefore, participants probably stopped preparatory activity before it could trigger overt behavior. Note that the main go-LRP of the hand $=$ gender task, which eventually triggered the overt responses, is temporally separated from the early activity in go- and no-go-waveform. If grammatical gender had been reliably retrieved at the time of the early LRP activations, one would expect that preparatory activity on go-trials would continuously increase from that moment on and develop into a full-blown LRP. The latter did not happen and in our view this suggests that early and late LRP activity in the hand $=$ gender task reflect different processes. As outlined above, we would propose that activity in the early time window relates to guessing grammatical gender on the basis of orthographic features, and activity in the late window relates to retrieving gender from the lexicon. We think that the suffix-checking strategy offers a viable explanation for the early LRP activity in the hand = gender task. Regarding the different timing of early activity in the go- and no-go-LRP, however, we must admit that we have no satisfying answer to offer and must leave the issue unresolved.

Taken together, the results from the no-go LRPs and the N2 effect provide evidence that semantic category is available earlier than grammatical gender for written words presented in isolation.

A general issue regarding the interpretation of our study might be the use of two explicit categorization tasks to measure the retrieval of grammatical gender and semantic category. Forster (1979) proposed that categorization tasks have no direct access to language processing, but depend on extra readout and decision processes, which co-determine the outcome for the dependent measures. This raises the question whether our findings actually reflect differences in the retrieval time for gender and semantic category or differences in those secondary task processes. In particular, if the semantic decisions in our experiment have a more direct access to semantic category representations than the gender decisions to grammatical gender representations, this would distort our view on the targeted retrieval time course. We cannot completely rule out such a possibility. One should consider, though, that we used ERP measures, which reflect task-related processes at an earlier time than conventional behavioral measures. Although our ERP measures are not immune to strategic influences, they might avoid some effects emerging at later response stages. Schiller, Münte, Horemans, and Jansma (2003) investigated phonological regularities in German gender classes and their influence on gender decisions. Although they found a significant effect for RTs in a simple go/no-go task, the peak latency of the inhibition-related N2 effect showed no reliable difference. It is undisputable that gender and semantic decisions contain some artificiality, but we would assume that participants have to rely on the representations underlying normal language processing to successfully carry out these tasks. There is now a considerable number of studies that have used the twochoice go/no-go paradigm to investigate time-course issues in language processing (Abdel Rahman \& Sommer, 2003; Abdel Rahman, Van Turennout, \& Levelt, 2003; Rodriguez-Fornells, Schmitt, Kutas, \& Münte, 2002; 
Schmitt, Rodriguez-Fornells, et al., 2001; Schmitt, Schiltz, Zaake, Kutas, \& Münte, 2001; Schmitt, Münte, \& Kutas, 2000; Van Turennout et al., 1997; Van Turennout, Hagoort, \& Brown, 1998). To summarize, drawing valid conclusions regarding the time course of gender and semantic retrieval from our results presupposes that gender decision and semantic decision have equivalent access to representations of grammatical gender and semantic category, respectively.

At the least, our experiment might be viewed as a control for potential modality effects in the study of Schmitt, Rodriguez-Fornells, et al. (2001), who compared the timing of semantics and grammatical gender with the same experimental technique in the auditory modality. For the N2 effect, they determined an onset difference of $95 \mathrm{msec}$ in favor of semantics and a peak latency difference of $69 \mathrm{msec}$ in favor of semantics. The possibility had existed that cues to semantic and syntactic information were located in temporally separate stretches of the speech signal, thereby biasing the retrieval order (cf. Van den Brink \& Hagoort, 2004; Hagoort, 2003). In that case, written words could have shown a different retrieval order, as all perceptual information becomes available at once with visual presentation. However, the timing of access to semantics and grammatical gender showed a similar profile for our written and their spoken stimuli.

Assuming the data of Schmitt, Rodriguez-Fornells, et al. (2001) and of the current study reflect the time course of gender and semantic category retrieval, they challenge the hypothesis that processing order in language comprehension is simply reversed in relation to that in speech production. Theoretical motivation for the reversal hypothesis comes from Levelt et al.'s (1999) claim that speech production and language comprehension share the semantic and grammatical representations for words. Furthermore, Levelt et al. and other speech production theories (cf. Levelt, 1999) assume that semantic processing precedes syntactic processing. Empirical evidence for this comes from Schmitt, Rodriguez-Fornells et al.'s tacit picture-naming experiment, where the N2 effect had an earlier onset and peak latency for semantics than for grammatical gender. According to the reversal hypothesis, language comprehension should proceed from syntactic to semantic processing. However, this is not what Schmitt, RodriguezFornells, et al. and we found.

To investigate this apparent contradiction, Roelofs (personal communication) implemented a simulation in his WEAVER model on the basis of our experiment. In this model, the presentation of a word provides the corresponding lemma node with input activation, which is then spreading to the appropriate gender node via a unidirectional connection (Figure 3; for general features of the model and the relation of speech production and language comprehension, cf. Roelofs, Meyer, \& Levelt, 1996; Roelofs, 1992). From the lemma, activation also

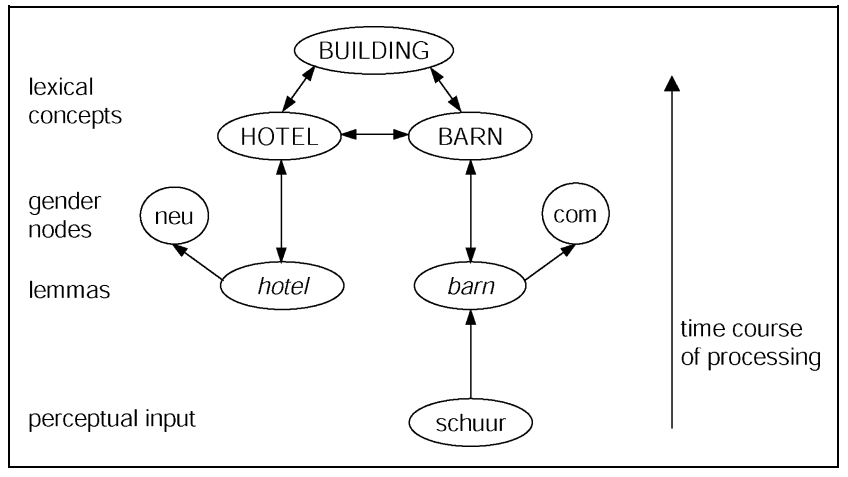

Figure 3. Sketch of the WEAVER model as adapted for language comprehension. Connections with one arrowhead are unidirectional and connections with two arrowheads are bidirectional (all excitatory) Activation starts to spread from the perceptual input to the corresponding lemma. From there, gender nodes (neu $=$ neuter gender, com $=$ common gender) and a corresponding concept node receive activation. The concept node can send activation back to its lemma and to superordinate concepts (building) and category coordinates (hotel).

spreads to the respective concept node, then to the corresponding semantic category node, but also to other category exemplars, which again feed activation to the semantic category. The connections from lemmas to concepts and among concepts are bidirectional. Although in the simulation, the gender nodes received the first portions of activation earlier than semantic category nodes, semantic category nodes reached the activation threshold set for final selection earlier than the gender nodes. According to Roelofs, this arises from the dynamic aspects of the model. Whereas gender nodes receive input from a lemma via one unidirectional connection, semantic category nodes are embedded in an extensive network with several inputs and bidirectional connections. The latter allows the reverberation of activation, which leads to an accelerated accumulation of activation for the semantic category nodes. The outcome of the simulation means that the hypothesis of shared semantic and syntactic representations for speech production and language comprehension is still a possibility and that we cannot refute it on the grounds of our timecourse results.

Our data show that there is a measurable time difference in the availability of semantic category and grammatical gender information. This excludes a serial discrete architecture where first retrieval of syntactic information has to finish before retrieval of semantic information can begin. The results leave room for a serial discrete system with retrieval of semantics before syntax as well as for a parallel system, with semantic retrieval being faster than syntactic retrieval (cf. Abdel Rahman \& Sommer, 2003). Roelofs' simulation indicates that in a parallel architecture the onset of access to semantic and syntactic representations might even have the opposite timing profile as final retrieval. 
Research on the details of accessing semantic and syntactic word properties in language comprehension has been sparse in the past. Although the fields of word recognition and sentence processing make reference to semantic and syntactic word properties, they often leave the specifics of their retrieval open. We believe our finding that semantic category information is available earlier than grammatical gender information when participants read single words contributes to a more complete picture of language comprehension. It puts restrictions on theories and models of language comprehension, especially on those that deal with the use of stored semantic and syntactic information.

In the sentence processing literature, there is a longlasting debate about when different kinds of lexical information contribute to the construction of a syntactic structure. Serial discrete models assume that this parsing process relies purely on syntactic lexical information at an early stage and takes semantic information into account only later (Friederici, 2002; Frazier, 1989; Forster, 1979). In contrast, constraint-based models assume that semantic and discourse information can exert an immediate influence (MacDonald et al., 1994; Trueswell \& Tanenhaus, 1994). Because we presented isolated words in our experiment, we cannot directly derive statements on sentence processing. In fact, from the point of view of serial discrete models, it might seem irrelevant when semantic and syntactic word properties become available. Time-course assumptions in such models rather describe when certain word properties get used in parsing and not when they are, in principle, available. However, for all we know about the incremental nature of language processing, the assumption that semantic information that is available earlier than syntactic lemma information will be used later in on-line sentence processing seems highly implausible. In contrast to serial discrete models, the constraint-based approach emphasizes that lexical processing, that is, the access to semantic and syntactic word properties, and the building of a sentence structure are intimately linked. All available lexical information of a word determines how the existing sentence and discourse structures change through the integration of the word, whereas the sentence and discourse structures can also have an impact on the activation of lexical information. Representatives of the constraint-based approach have made no commitments to the time course of access for different word properties, focusing on the interactive character of lexical and sentence processing. Nonetheless, the finding that during the presentation of isolated words certain aspects of lexical semantic information are available earlier than certain aspects of syntactic information, might mean that lexical semantic information influences sentence structure building earlier than lexical syntactic information. Just as much, a biasing sentence or discourse context might alter the time course in which lexical semantic and syntactic information become available.
To know when certain word properties are available may also put constraints on certain word recognition models that assume interactions among orthographic, phonological, semantic, and syntactic representations of a word (Plaut, McClelland, Seidenberg, \& Patterson, 1996; Van Orden, Pennington, \& Stone, 1990). Such models would have to accommodate findings on the time course of availability of semantic and syntactic word properties and be able to mirror these findings in simulations. This obviously concerns the dynamics of the semantic and syntactic representations, but because of the extensive interactivity in these models, there may also be consequences for the dynamics of orthographical and phonological representations. Plaut et al. (1996, simulation 4), for example, have shown that an orthography-to-phonology mapping in a parallel distributed processing network is learned in different ways, depending on the presence or absence of an orthographyto-semantics-to-phonology pathway. In analogy to that, different patterns of access to semantic and syntactic representations of a word could have distinctive effects on the processing in the orthographical and phonological parts of the word recognition system.

Our study probed the processes of semantic and syntactic access in language comprehension, which lie at the junction of word recognition and sentence processing. Using electrophysiological recordings to monitor these covert processes with high temporal resolution, we could establish that semantic information is available earlier than syntactic information. How the differential temporal availability of semantic and syntactic word properties fits into the dynamics of word identification and sentence processing remains a topic for future research.

\section{METHODS}

\section{Participants}

Thirty-two native speakers of Dutch (27 women) participated in the experiment, with age being on average 21.3 years and ranging from 17 to 27 years. All participants were right-handed and had normal or correctedto-normal vision. None of them suffered from a neurological impairment, had experienced a neurological trauma, or was taking neuroleptics. The participants received payment for taking part in the experiment.

\section{Materials}

Words from the eight semantic categories building, consumable (food and beverages), landscape formation, animal, part of a house, clothing, weapon, and body part served as stimuli. For the semantic decision task, we constructed eight different category pairings to allow for binary decisions (see Table 2). Each category contributed common and neuter gender words. In order to 
Table 2. First and Second Sets of Category Pairings for the Semantic Decision

\begin{tabular}{lll}
\hline Set 1 & & \\
$\begin{array}{l}\text { 1. building } \\
\text { 2. building }\end{array}$ & vs. & consumable \\
3. landscape formation & vs. & consumable \\
4. landscape formation & vs. & animal \\
Set 2 & & \\
5. part of a house & & clothing \\
6. part of a house & vs. & body part \\
7. weapon & vs. & clothing \\
8. weapon & vs. & body part \\
\hline
\end{tabular}

achieve an approximately equal number of common and neuter gender words within each semantic category, diminutives were included in the item set. In Dutch, diminutives are formed by attaching a suffix to a noun and automatically carry neuter gender, regardless of the gender of the base word. Each category contained 12 or 14 monomorphemic common gender targets and usually an equal number of neuter gender targets (never less than $45 \%$ neuter gender nouns; see Table 3 for details). At least half of the neuter gender targets within a category were monomorphemic and had intrinsic neuter gender, whereas the rest were diminutives. Within a category pairing, the distributions of word length in letters and lemma frequency substantially overlapped for the two semantic categories and the two gender classes (see Table 3). Six additional items from each category (three from each gender class) served as practice trials before experimental blocks and another extra item served as warm-up trial in the experimental blocks. For a practice block at the beginning of a session, we used words from the categories musical instrument and plant.

\section{Procedure}

Participants were seated in a dimly lit sound-attenuating booth, facing a computer screen. They rested their arms on the arm rests of the chair and held the left and right index fingers on response buttons, inserted in the arm rests. A trial started with the presentation of a fixation cross in the middle of the screen for 2000 msec. Next the screen turned blank for $1000 \mathrm{msec}$ and then a word appeared on the screen for $1000 \mathrm{msec}$. After disappearance of the word, the screen stayed blank for $2050 \mathrm{msec}$ and a new trial began. Words were presented in white lowercase Arial letters against dark background. Viewing distance was approximately $100 \mathrm{~cm}$, the visual angle for the longest word was about $2.4^{\circ}$ horizontally and $0.4^{\circ}$ vertically. Participants performed a two-choice go/no-go task. One half of the participants received instructions in which grammatical gender determined response hand (left/right) and semantic category determined response execution (go/no-go). The other half received instructions that mapped semantic category on response hand and grammatical gender on response execution (see Table 1 for examples). Participants were asked to keep

Table 3. Statistics of Item Set, Split Up for Semantic Categories and Common (com) and Neuter (neu) Gender Nouns

\begin{tabular}{|c|c|c|c|c|c|c|}
\hline \multirow[b]{2}{*}{ Semantic Category } & \multicolumn{2}{|c|}{ Number of Items } & \multicolumn{2}{|c|}{ Length in Letters Mean (Min-Max) } & \multicolumn{2}{|c|}{ Lemma Frequency ${ }^{a}$} \\
\hline & com & Intrinsic neu + dim $^{b}$ & com & $n e u^{c}$ & com & $n e u^{c}$ \\
\hline Building & 14 & $7+7$ & $5.4(3-8)$ & $6.1(3-8)$ & 36.1 & 38.8 \\
\hline Consumable & 14 & $7+7$ & $5.0(3-8)$ & $5.4(4-8)$ & 36.2 & 28.1 \\
\hline Landscape formation & 14 & $7+6$ & $5.8(3-8)$ & $5.5(3-7)$ & 27.9 & 22.5 \\
\hline Animal & 14 & $7+7$ & $5.4(3-8)$ & $5.7(4-8)$ & 18.2 & 30.2 \\
\hline Part of a house & 12 & $6+4$ & $4.9(3-7)$ & $5.9(3-8)$ & 96.7 & 48.9 \\
\hline Clothing & 12 & $6+6$ & $5.2(3-7)$ & $5.8(4-8)$ & 15.6 & 13.9 \\
\hline Weapon & 12 & $6+4$ & $5.2(3-8)$ & $6.0(3-8)$ & 20.3 & 16.9 \\
\hline Body part & 12 & $6+6$ & $4.8(3-8)$ & $5.3(3-8)$ & 53.3 & 133.1 \\
\hline Sum/average ${ }^{\mathrm{d}}$ & 104 & $52+47$ & 5.2 & 5.7 & 37.4 & 41.1 \\
\hline
\end{tabular}


their arms relaxed and not to blink or move their eyes, except for the period when the fixation cross was on the screen.

There was a practice block comprising 96 trials at the beginning of each session, followed by eight experimental blocks (Table 2). Each block started with an instruction given via the screen, indicating for which class of words participants were supposed to press the left or right button and for which class of words the movement had to be executed or not. Examples were provided for all response possibilities (left-go, left-no-go, right-go, right-no-go). A series of 12 practice trials followed the instruction. Experimental blocks contained 46 to 56 stimuli, depending on the semantic categories involved. Within blocks, items occurred in a pseudorandomized order, with the restriction that maximally three items of a given grammatical gender or semantic category would appear in succession. No more than two participants received the same randomization. For a given participant, the mapping of grammatical gender onto response dimension (left/right or go/no-go) was the same for all blocks from the category pairings of Set 1 (Table 2) and the reversed mapping for all blocks from the category pairings of Set 2. The response mapping of grammatical gender within these sets of category pairings was balanced across participants. The mapping of a semantic category onto response dimensions was constant for a given participant, while it was balanced across participants. The required response for an item stayed the same across repetition. The order of blocks was randomized with the following restrictions: After the first experimental block, a block from the opposite set of category pairings occurred, so that the grammatical gender mapping changed. Furthermore, blocks containing the same semantic category were separated by two blocks containing other semantic categories. This led to an ABBAABBA structure of block order, where A and $B$ represent blocks from a particular set of category pairings.

\section{Apparatus and Recordings}

The EEG was recorded at 26 sites on the scalp, with reference to the left mastoid. These sites represent a selection of electrode slots in the Easy-Cap Montage No. 10 as provided by Falk Minow Services (for theta/phi coordinates see: Theta/phi-coordinates of equidistant montage no. 10, n.d.). The six midline sites with the numbers 35, 20, 2, 1, 14, and 43 correspond to the positions $\mathrm{Fpz}, \mathrm{AFz}, \mathrm{FCz}, \mathrm{Cz}, \mathrm{Pz}$, and $\mathrm{Oz}$ of the $10 \%$ system of the American Electroencephalographic Society (1991). The remaining electrodes were placed laterally over symmetrical positions: frontal (in pairs of corresponding electrodes: 49, 37; 50, 36; 33, 22; 34, 21), frontocentral $(18,10 ; 7,3 ; 17,11 ; 6,4)$, and occipital $(45,41 ; 44,42)$. We used the electrode pairs $18 / 10$ and $7 / 3$ in the computation of the LRP. Electrode pair 18/10 was approximately
$6 \mathrm{~cm}$ lateral and $3 \mathrm{~cm}$ anterior to $\mathrm{Cz}$, whereas electrode pair $7 / 3$ was approximately $3 \mathrm{~cm}$ lateral and $2 \mathrm{~cm}$ anterior to $\mathrm{Cz}$. In order to control the quality of the left mastoid as neutral reference, an additional electrode was attached to the right mastoid, referenced to the left mastoid. A ground electrode was placed on the forehead. Blinks and vertical eye movements were recorded bipolarly using electrodes situated above and below the left eye. Horizontal eye movements were monitored via a bipolar montage of electrodes positioned external to the left and right outer canthus of each eye. The EMG of the left and right forearm flexors was recorded bipolarly with electrodes placed following the recommendations of Lippold (1967). Ag/AgCl electrodes were used for all recordings. Electrode impedance was kept below $3 \mathrm{k} \Omega$ for the EEG recording, below $5 \mathrm{k} \Omega$ for the electrooculogram (EOG) recording, and below $10 \mathrm{k} \Omega$ for the EMG recording. The signals were amplified by a Neuroscan SynAmp amplifier and data acquisition occurred via Scan 4.1 Software from Neuroscan. For all recordings, a time constant of $8 \mathrm{sec}$ was set. The highfrequency cutoff was $30 \mathrm{~Hz}$ for EEG and EOG recordings and $100 \mathrm{~Hz}$ for the EMG recording. Digitization of the signals took place on-line with a sampling frequency of $500 \mathrm{~Hz}$. Sampling started $150 \mathrm{msec}$ before word onset and continued for an epoch of $2100 \mathrm{msec}$.

The 150-msec period before word onset served as baseline and its average voltage per trial and electrode was subtracted from the respective waveforms. Artifact control occurred for epochs from $150 \mathrm{msec}$ before word onset until $1500 \mathrm{msec}$ after word onset. The EMG was visually inspected for discernible activity on no-go-trials and activity of the wrong response hand on go-trials. EOG and scalp electrodes were controlled for eye movement artifacts, amplifier blocking, and amplitudes exceeding $75 \mu \mathrm{V}$ above or below baseline. Behavioral errors were coded for no-go-trials in which a pushbutton response was registered and for go-trials where the wrong push-button was pressed or no response was registered within 1500 msec after stimulus appearance. For each participant, there were at least 35 trials left per response hand and go/no-go condition.

\section{Acknowledgments}

This research was supported by a PhD grant to $\mathrm{O}$. M. from the Max-Planck-Gesellschaff zur Förderung der Wissenschaften, München, Germany.

We thank Rasha Abdel Rahman, Michael Coles, Ardi Roelofs, and Miranda van Turennout for their intellectual input; René de Bruin, John Nagengast, and Johan Weustink for technical support; and Valesca Kooijman, Karen de Waele, Christopher Miller, Marieke van der Linden, and Jelle van Dijk for help in acquiring the ERP data.

Reprint requests should be sent to Oliver Müller, F.C. Donders Centre for Cognitive Neuroimaging, P.O. Box 9101, 6500 HB Nijmegen, The Netherlands, or via e-mail: oliver.muller@ fcdonders.ru.nl. 


\section{Notes}

1. Activation flow between the concept and lemma level is assumed to be cascaded and bidirectional, so that periods of activation in the two layers may overlap.

2. We adopted the formula proposed by De Jong et al. (1988): $\mathrm{LRP}=$ Average $(\mathrm{L}-\mathrm{R})_{\text {righthand }}-$ Average $(\mathrm{L}-\mathrm{R})_{\text {lefthand }}$ Where $L$ and $R$ represent electrodes seated above the left and right motor cortices, respectively. De Jong et al. refer to it as the corrected motor asymmetry, but the common label nowadays is LRP. This formula is equivalent to the proposal of Coles et al. (1988), with the exception that the latter results in a LRP of half the amplitude.

3. We also applied a jackknifing procedure, which estimates ERP onset latencies on the basis of grand averages and uses a conventional ANOVA, plus some simple adjustment of the $F$ ratio (Ulrich \& Miller, 2001). We set an absolute onset criterion of $-0.4 \mu \mathrm{V}$, surveying the period from 350 to $600 \mathrm{msec}$. The estimated onset latencies were similar to the ones from the $t$-test procedure, $427 \mathrm{msec}$ for the hand $=$ semantics task and 399 for the hand = gender task. This difference was not significant in a between-subject ANOVA with the factor Task [adjusted $F(1,30)<1$ ]. Furthermore, 14 participants had additionally performed a second task for exploratory purposes. Half of them had started with the semantics = hand task and then performed the gender $=$ hand task, whereas the others had followed the opposite task order (only first task data had entered the between-subjects analysis reported above). For these counterbalanced within-subjects data, serial $t$ tests against baseline resulted in a go-LRP onset latency of $450 \mathrm{msec}$ for the semantics $=$ hand task and $470 \mathrm{msec}$ for the gender = hand task. A direct comparison of the go-waveforms with serial $t$ tests and the jackknife procedure showed no significant onset difference.

4. We performed univariate $F$ tests in our analyses and corrected violations of sphericity for repeated-measures factors by adjusting $d f \mathrm{~s}$ with the Greenhouse-Geisser epsilon. When applicable, we report the uncorrected $d f \mathrm{~s}$ and $M S E$, followed by the corrected $p$ value and the $\varepsilon$ value.

\section{REFERENCES}

Abdel Rahman, R., \& Sommer, W. (2003). Does phonological encoding in speech production always follow the retrieval of semantic knowledge? Electrophysiological evidence for parallel processing. Cognitive Brain Research, 16, 372-382.

Abdel Rahman, R., Van Turennout, M., \& Levelt, W. J. M. (2003). Phonological encoding is not contingent on semantic feature retrieval: An electrophysiological study on object naming. Journal of Experimental Psychology: Learning, Memory, and Cognition, 29, 850-860.

Allopenna, P. D., Magnuson, J. S., \& Tanenhaus, M. K. (1998). Tracking the time course of spoken word recognition using eye movements: Evidence for continuous mapping models. Journal of Memory and Language, 38, 419-439.

American Electroencephalographic Society. (1991). Guidelines for standard electrode position nomenclature. Journal of Clinical Neurophysiology, 8, 200-202.

Balota, D. A. (1994). Visual word recognition: The journey from features to meaning. In M. A. Gernsbacher (Ed.), Handbook of psycholinguistics (pp. 303-358). San Diego, CA: Academic Press.

Caramazza, A. (1997). How many levels of processing are there in lexical access? Cognitive Neuropsychology, 14, 177-208.

CELEX Dutch database (Release N31) [On-line]. (1990). Retrieved 18 April 2005 from www.mpi.nl/world/celex.
Coles, M. G. H., Gratton, G., \& Donchin, E. (1988). Detecting early communication: Using measures of movement-related potentials to illuminate human information processing. Biological Psychology, 26, 69-89.

Corbett, G. G. (1991). Gender. Cambridge: Cambridge University Press.

De Jong, R., Wierda, M., Mulder, G., \& Mulder, L. J. M. (1988). Use of partial stimulus information in response processing. Journal of Experimental Psychology: Human Perception and Performance, 14, 682-692.

Donaldson, B. C. (1987). Dutch reference grammar (3rd ed.). Leiden: Nijhoff.

Eimer, M. (1993). Effects of attention and stimulus probability on ERPs in a go/nogo task. Biological Psychology, 35, 123-138.

Falkenstein, M., Hoormann, J., \& Hohnsbein, J. (1999). ERP components in go/nogo tasks and their relation to inhibition. Acta Psychologica, 101, 267-291.

Forster, K. I. (1979). Levels of processing and the structure of the language processor. In W. E. Cooper \& E. C. T. Walker (Eds.), Sentence processing: Psycholinguistic studies presented to Merrill Garrett (pp. 27-85). Hillsdale, NJ: Erlbaum.

Frazier, L. (1989). Against lexical generation of syntax. In W. D. Marslen-Wilson (Ed.), Lexical representation and process (pp. 505-528). Cambridge: MIT Press.

Friederici, A. D. (2002). Towards a neural basis of auditory sentence processing. Trends in Cognitive Sciences, 6, 78-84.

Friederici, A. D., \& Jacobsen, T. (1999). Processing grammatical gender during language comprehension. Journal of Psycholinguistic Research, 28, 467-484.

Friederici, A. D., Pfeifer, E., \& Hahne, A. (1993). Event-related brain potentials during natural speech processing: Effects of semantic, morphological, and syntactic processing. Cognitive Brain Research, 1, 183-192.

Gemba, H., \& Sasaki, K. (1989). Potential related to no-go reaction of go/no-go hand movement task with color discrimination in human. Neuroscience Letters, 101, 263-268.

Hagoort, P. (2003). How the brain solves the binding problem for language: A neurocomputational model of syntactic processing. Neuroimage, 20, S18-S29.

Hahne, A., \& Jescheniak, J. D. (2001). What's left if the Jabberwock gets the semantics? An ERP investigation into semantic and syntactic processes during auditory sentence comprehension. Cognitive Brain Research, 11, 199-212.

Jodo, E., \& Kayama, Y. (1992). Relation of a negative ERP component to response inhibition in a go/nogo task. Electroencephalography and Clinical Neurophysiology, 82, 477-482.

Kok, A. (1986). Effects of degradation of visual stimuli on components of the event-related potential (ERP) in go/nogo reaction tasks. Biological Psychology, 23, 21-38.

Kornhuber, H. H., \& Deecke, L. (1965). Hirnpotentialänderungen bei Willkürbewegungen und passiven Bewegungen des Menschen: Bereitschaftspotential und reafferente Potentiale [Brain potential changes associated with voluntary and passive movements in humans: Readiness potential and reafferent potentials]. Pflügers Archive, 284, 1-17.

Kutas, M., \& Donchin, E. (1980). Preparation to respond as manifested by movement-related brain potentials. Brain Research, 202, 95-115.

Kutas, M., \& Van Petten, C. K. (1994). Psycholinguistics electrified. In M. A. Gernsbacher (Ed.), Handbook of psycholinguistics (pp. 83-143). San Diego, CA: Academic Press. 
Levelt, W. J. M. (1999). Models of word production. Trends in Cognitive Sciences, 3, 223-232.

Levelt, W. J. M., Roelofs, A., \& Meyer, A. S. (1999). A theory of lexical access in speech production. Behavioral and Brain Sciences, 22, 1-75.

Lippold, O. C. J. (1967). Electromyography. In P. H. Venables \& I. Martin (Eds.), A manual of psychophysiological methods (pp. 245-297). Amsterdam: North-Holland Publishing.

MacDonald, M. C., Pearlmutter, N. J., \& Seidenberg, M. S. (1994). Lexical nature of syntactic ambiguity resolution. Psychological Review, 101, 676-703.

Marslen-Wilson, W. D. (1987). Functional parallelism in spoken word recognition. Cognition, 25, 71-102.

McClelland, J. L., \& Elman, J. L. (1986). The TRACE model of speech perception. Cognitive Psychology, 18, 1-86.

Miller, J., \& Hackley, S. A. (1992). Electrophysiological evidence for temporal overlap among contingent mental processes. Journal of Experimental Psychology: General, 121, 195-209.

Norris, D. (1994). Shortlist: A connectionist model of continuous speech recognition. Cognition, 52, 189-234.

Osman, A., Bashore, T. R., Coles, M. G. H., Donchin, E., \& Meyer, D. E. (1992). On the transmission of partial information: Inferences from movement-related brain potentials. Journal of Experimental Psychology: Human Perception and Performance, 18, 217-232.

Pfefferbaum, A., Ford, J. M., Weller, B. J., \& Kopell, B. S. (1985). ERPs to response production and inhibition. Electroencephalography and Clinical Neurophysiology, 60, 423-434.

Plaut, D. C., McClelland, J. L., Seidenberg, M. S., \& Patterson, K. (1996). Understanding normal and impaired word reading: Computational principles in quasi-regular domains. Psychological Review, 103, 56-115.

Radeau, M., Morais, J., Mousty, P., Saerens, M., \& Bertelson, P. (1992). A listener's investigation of printed word processing. Journal of Experimental Psychology: Human Perception and Performance, 18, 861-871.

Rastle, K., \& Coltheart, M. (1999). Serial and strategic effects in reading aloud. Journal of Experimental Psychology: Human Perception and Performance, 25, 482-503.

Rodriguez-Fornells, A., Schmitt, B. M., Kutas, M., \& Münte, T. F. (2002). Electrophysiological estimates of the time course of semantic and phonological encoding during listening and naming. Neuropsychologia, 40, 778-787.

Roelofs, A. (1992). A spreading-activation theory of lemma retrieval in speaking. Cognition, 42, 107-142.

Roelofs, A., Meyer, A. S., \& Levelt, W. J. M. (1996). Interaction between semantic and orthographic factors in conceptually driven naming: Comment on Starreveld and La Heij (1995). Journal of Experimental Psychology: Learning, Memory, and Cognition, 22, 246-251.

Schiller, N. O., Münte, T. F., Horemans, I., \& Jansma, B. M. (2003). The influence of semantic and phonological factors on syntactic decisions: An event-related brain potential study. Psychophysiology, 40, 869-877.

Schmitt, B. M., Münte, T. F., \& Kutas, M. (2000).

Electrophysiological estimates of the time course of semantic and phonological encoding during implicit picture naming. Psychophysiology, 37, 473-484.

Schmitt, B. M., Rodriguez-Fornells, A., Kutas, M., \& Münte, T. F. (2001). Electrophysiological estimates of semantic and syntactic information access during tacit picture naming and listening to words. Neuroscience Research, 41, 293-298.

Schmitt, B. M., Schiltz, K., Zaake, W., Kutas, M., \& Münte, T. F. (2001). An electrophysiological analysis of the time course of conceptual and syntactic encoding during tacit picture naming. Journal of Cognitive Neuroscience, 13, 510-522.

Schriefers, H., \& Teruel, E. (2000). Journal of Experimental Psychology: Learning, Memory, and Cognition, 26, 1368-1377.

Simson, R., Vaughan, H. G., \& Ritter, W. (1977). The scalp topography of potentials in auditory and visual go/nogo tasks. Electroencephalography and Clinical Neurophysiology, 43, 864-875.

Smid, H. G. O. M., Mulder, G., Mulder, L. J. M., \& Brands, G. J. (1992). A psychophysiological study of the use of partial information in stimulus-response translation. Journal of Experimental Psychology: Human Perception and Performance, 18, 1101-1119.

Theta/phi-coordinates of equidistant montage no. 10. (n.d.). Retrieved 3 February 2005 from www.easycap.de/easycap/e/ downloads/M10_ThetaPhi.htm.

Thorpe, S., Fize, D., \& Marlot, C. (1996). Speed of processing in the human visual system. Nature, 381, 520-522.

Trueswell, J. C., \& Tanenhaus, M. K. (1994). Toward a constraint-based lexicalist approach to syntactic ambiguity resolution. In C. Clifton, L. Frazier, \& K. Rayner (Eds.), Perspectives on sentence processing (pp. 155-179). Hillsdale, NJ: Erlbaum.

Ulrich, R., \& Miller, J. (2001). Using the jackknife-based scoring method for measuring LRP onset effects in factorial designs. Psychophysiology, 38, 816-827.

Van Berkum, J. J. A. (1996). The psycholinguistics of grammatical gender: Studies in language comprebension and production. Nijmegen: Nijmegen University Press.

Van den Brink, D., \& Hagoort, P. (2004). The influence of semantic and syntactic context constraints on lexical selection and integration in spoken-word comprehension as revealed by ERPs. Journal of Cognitive Neuroscience, 16, 1068-1084.

Van Orden, G. C., Pennington, B. F., \& Stone, G. O. (1990). Word identification in reading and the promise of subsymbolic psycholinguistics. Psychological Review, 97, 488-522.

Van Turennout, M., Hagoort, P., \& Brown, C. M. (1997). Electrophysiological evidence on the time course of semantic and phonological processes in speech production. Journal of Experimental Psychology: Learning, Memory, and Cognition, 23, 787-806.

Van Turennout, M., Hagoort, P., \& Brown, C. M. (1998). Brain activity during speaking: From syntax to phonology in 40 milliseconds. Science, 280, 572-574.

Zorzi, M. (2000). Serial processing in reading aloud: No challenge for a parallel model. Journal of Experimental Psychology: Human Perception and Performance, 26, 847-856. 